\title{
FMCW Radar with Broadband Communication Capability
}

\author{
Peli Barrenechea, Frans Elferink and Johan Janssen \\ TNO Defence, Security and Safety \\ P.O. 96864, 2509JG The Hague, The Netherlands \\ \{peli.barrenechea@tno.nl, frans.elferink@tno.nl, johan.janssen@tno.nl\}
}

\begin{abstract}
The use of amplitude modulation to encode information onto an FMCW radar signal is proposed in this paper. This new technique, that has been named AM-FMCW communicating radar, provides a new channel for broadband communication by reusing the radar frequencies and without introducing any distortion in the radar functionality.
\end{abstract}

\section{INTRODUCTION}

The data bandwidth demand for new applications increases everyday. This makes the information capacity available really scarce, and thus, expensive. At the same time, new radar networks are being created for civil applications. Reusing the radar frequencies to send information as well increases the available data bandwidth and creates new communication channels. Adding information to radar signals, by using different kind of modulation schemes or time division techniques, has been studied since many years. In this paper, the new technique of using Amplitude Modulation (AM) to encode data onto a Frequency Modulated Continuous Wave (FMCW) radar signal is presented. This technique has been named AM-FMCW communicating radar. The proposed solution combines data communication and radar simultaneously in the same architecture.

This paper is organized as follows. In Section II FMCW is shortly introduced and a system overview is given. The theoretical underpinning of the proposed methods is given in Section III. The validate them simulations are performed, the results are given in Section IV. Finally, Section V states the conclusions.

\section{THE AM-FMCW SYSTEM OVERVIEW}

FMCW radar is a popular technology that nowadays is being employed very often in modern radar applications, mainly because of its good performance, low cost and low power consumption. Adding data to the FMCW signal allows the development of new application areas. The combination of radar and data communication in a single signal seems to be especially interesting for actual infrastructures with a large number of FMCW radars, such as traffic radar networks [2]. Adding data to the radar signal requires only a simple modification in the form of an amplitude modulator. Recovering the data encoded in the amplitude is more complicated. A general representation of the AM-FMCW system is shown in Fig. 1.

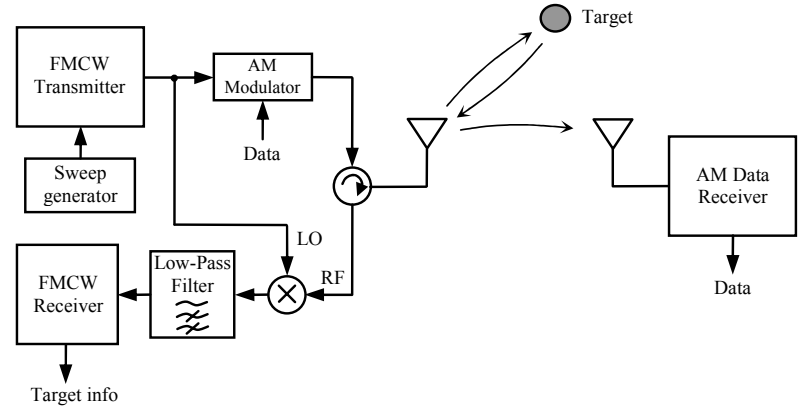

Fig. 1 AM-FMCW system overview

\section{THEORETICAL ANALYSIS}

FMCW radars utilize a continuous wave modulated in frequency by a repetitive linear frequency sweep, which generally has a sawtooth shape (frequency against time) as shown in Fig. 2.

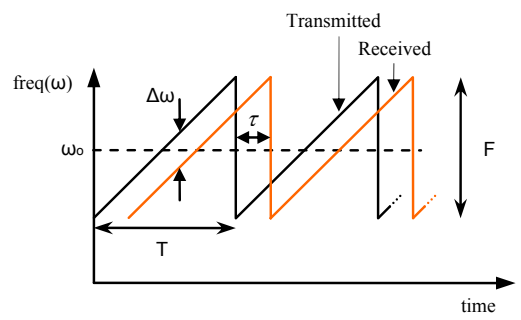

Fig. 2 Transmitted and received FMCW frequency sweeps

The transmitted radar signal is reflected by the target and is received by the radar antenna. The frequency difference between the transmitted and received signals, represented as $\Delta \omega$ in Fig. 2, is a result of the propagation time to the target and back $(\tau)$, which is also depicted in Fig. 2. By mixing and low-pass filtering the transmitted and received signals, as shown in Fig. 1, this frequency difference $(\Delta \omega)$ can be easily measured. The frequency difference is linear proportional to the distance between the radar and the target. Repeating this operation several times, the Doppler frequency can be measured as well, which directly gives the radial velocity of the target [1]. 


\section{A. AM-FMCW analytical study}

The transmitted FMCW signal, for the case of a linear sweep as presented in Fig. 2, is written as:

$$
x_{F M C W}(t)=A_{F M C W} \cos \left(\omega_{o} t+\int_{0}^{t} \Delta_{s} \theta \cdot d \theta\right)
$$

where $\Delta_{s}=F / T$, and is defined as the slope of the frequency sweep.

If any data or other information, $d(t)$, is encoded on the FMCW signal using amplitude modulation, the AM-FMCW signal results:

$$
x_{F M C W}^{A M}(t)=(1+m \cdot d(t)) \cdot x_{F M C W}(t)
$$

in which $m$ represents the amplitude modulation index $(0<m<1)$.

When the transmitted AM-FMCW signal is reflected by a target, the signal coming back to the radar system has the same shape, but attenuated in amplitude and delayed in time because of the propagation time to the target and back $(\tau)$. Thus, the received signal becomes:

$$
y_{F M C W}^{A M}(t)=A_{A T T}(1+m d(t-\tau)) \cdot x_{F M C W}(t-\tau)
$$

The transmitted signal without AM and the received signal are mixed and low-pass filtered (LP) in the FMCW radar receiver in order to get the target information:

$$
\begin{aligned}
& M_{F M C W}^{A M}(t)=L P\left[x_{F M C W}(t) \cdot y_{F M C W}^{A M}(t)\right]= \\
& =\frac{1}{2} A_{F M C W}^{2} A_{A T T} \cos \theta_{o} \cdot(1+m d(t-\tau))
\end{aligned}
$$

where $\theta_{o}=\omega_{o} \tau-\frac{\Delta_{s}}{2} \tau^{2}+\Delta_{s} \tau \cdot t$. The propagation time $\tau$ to the target and back is defined as:

$$
\tau=\frac{2}{c}\left(d_{o}-v_{o} \cdot t\right)
$$

where $v_{o}$ is the velocity of a target approaching to the radar from a distance $d_{o}$. The first term of equation 4 , corresponds to the target information in a usual FMCW radar: in this case the target produces one frequency component at $\Delta \omega=\Delta_{s} \tau$. The second part appears due to the mixing between the target information and the amplitude modulation added, and introduces mixing components at $\Delta \omega \pm \omega_{d}$, where $\omega_{d}$ is the frequency of the data signal carrier. The amplitudes of these components are proportional to the amplitude modulation index $(m)$.

\section{B. Considerations on the AM-FMCW Radar}

According to the previous equations, it can be said that theoretically the FMCW target information remains in the mixed signal, although new frequency components appear due to the amplitude modulation added to the radar signal. When a relatively low value for $\omega_{d}$ (the frequency of the data signal carrier) compared to the maximum expected target frequency is chosen, the new components will appear around the target frequency peak, as shown in Fig. 3. This may clearly degrade the radar performance because these new components could be confused with other possible targets.

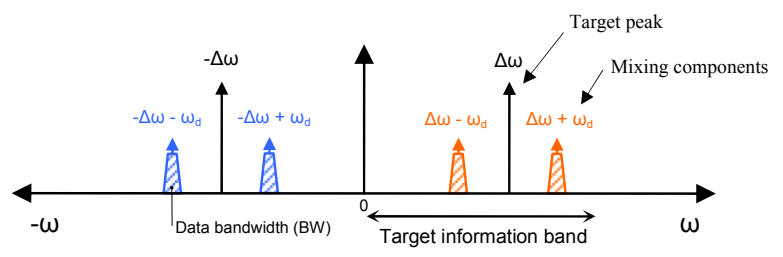

Fig. 3 Mixed signal spectrum when $\omega_{d}$ is low

To avoid this, the new components have to be placed out of the target information frequency band to avoid disturbing the radar functionality. Therefore, if $\omega_{d}$ is chosen high enough, the mixing components will appear far away from the target information band, as shows Fig. 4, and can be easily filtered out. In this case, the radar performance will not be degraded at all.

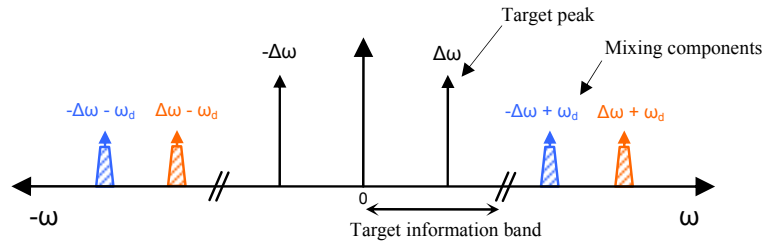

Fig. 4 Mixed signal spectrum when $\omega_{d}$ is high

Thus, on the AM-FMCW radar side, to theoretically ensure that the mixing components due to the amplitude modulation do not affect the radar performance, the frequency of the data signal carrier has to fulfil the equation:

$$
\omega_{d}>2 \cdot \Delta \omega_{\max }+\frac{B W}{2}
$$

where $\Delta \omega_{\max }$ is the frequency of the component produced by a target detected in the maximum range measurable by the FMCW radar, and $B W$ is the data signal bandwidth.

When applying equation 6 and placing a low-pass filter just after the mixer in the receiving part, as shown in Fig.1, the FMCW radar receiver processing remains the same [4] as it would be without amplitude modulated information.

\section{Considerations on the AM Data Receiver}

The AM data receiver is the destination of the information sent by the AM-FMCW Communicating Radar and is 
depicted in the right hand side of Fig. 1. It has to extract the AM information from the received radar signal.

In the AM-FMCW signal, the data is spread over the FMCW sweep frequencies, which could be easily over some hundred $\mathrm{MHz}$, while the information signal bandwidth is in the order of hundreds $\mathrm{kHz}$ or some $\mathrm{MHz}$. Thus, two problems emerge due to the lack of band limitation:

- The information is affected by noise, as it is proportional to the signal bandwidth.

- The amplitude information recovery is difficult. For instance, an envelope detector with such a broad bandwidth is hardly achievable.

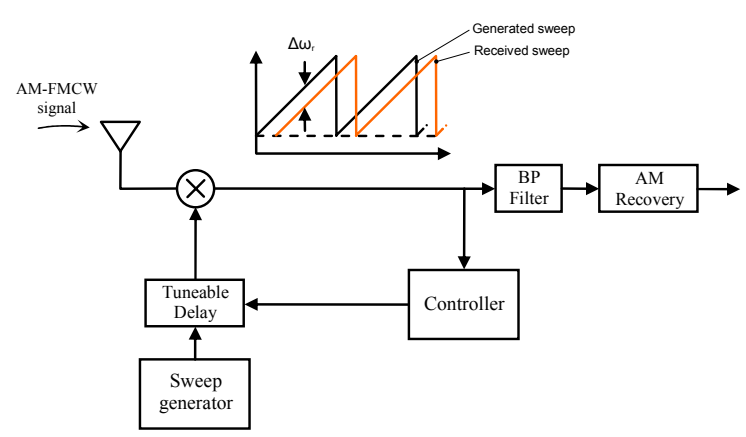

Fig. 5 AM data receiver structure

Fig. 5 represents a solution for the AM information recovery, in which the needed band limitation is accomplished. Basically, it mixes the received AM-FMCW signal with a generated sweep with the same characteristic as the one of the FMCW radar. Then, a loop structure is utilized in combination with a controller that acts on the tuneable delay to synchronize the received and the generated sweeps. The delay is adjusted until the frequency difference $\left(\Delta \omega_{r}\right)$ reaches the desired intermediate frequency ( $I F$ ). When this condition is achieved, $\Delta \omega_{r}=I F$, the loop gets locked, and in the mixer output spectrum one intermediate component will appear plus two data side bands at $I F \pm \omega_{d}$ (due to the mixing between $I F$ and the data signal), as shows Fig 6 . Then, by band-pass filtering one of the side bands and simple demodulation the data is first band limited and later recovered.

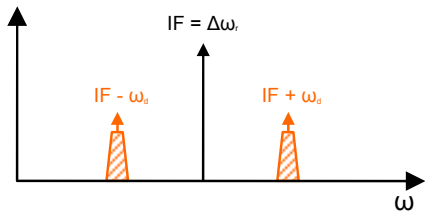

Fig. 6 Mixer output spectrum when loop locked

\section{SiMULATION RESULTS}

A practical FMCW radar for general purpose but including the amplitude modulated information has been simulated. The parameters used for the FMCW frequency sweep are shown in
Fig. 7. It is assumed that this general purpose FMCW radar is utilized to measure ranges up to 200 meters, resulting in target beat frequencies in the range FFT as high as $5 \mathrm{MHz}$. Thus, taking into account equation 6 , which defines the minimum AM frequency carrier in relation with the maximum measurable target range, and assuming AM information with a bandwidth of $1 \mathrm{MHz}$, it can be written that $\omega_{d}>2 \pi \cdot 10,5 \mathrm{MHz}$.

In order to avoid working on the edge of the condition and relax the receiver low-pass filter characteristic (Fig. 1), it was decided to use $\omega_{d}=2 \pi \cdot 20 \mathrm{MHz}$. Besides, a $10 \%$ amplitude modulation index was employed.

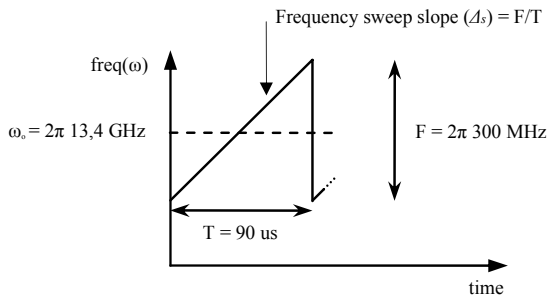

Fig. 7 FMCW frequency sweep simulation parameters

\section{A. AM-FMCW Radar Transmitter}

As explained before, the amplitude information is introduced in the FMCW signal on the radar transmitter side, where an AM modulator is placed. Fig. 8 shows the difference in the linear frequency modulated radar signal in time before and after the AM modulator.

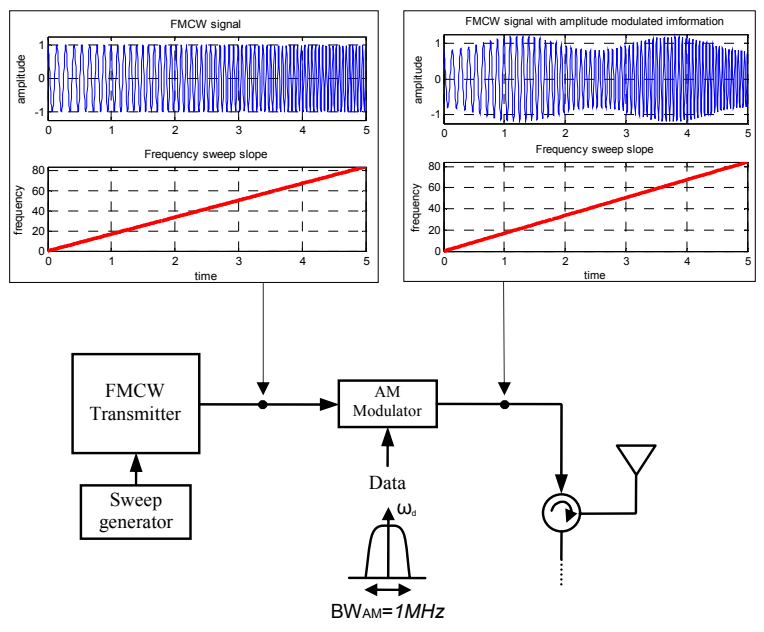

Fig. 8 AM-FMCW transmitter

\section{B. AM-FMCW Radar Receiver}

As already mentioned, the FMCW radar receiver processing remains the same, as the mixing components due to the AM information introduced can be filtered out with a low-pass filter. As shown in Fig. 1, it is placed just after the 
mixing between the received signal reflected by the target and the FMCW transmitted signal.

Fig. 9 shows the spectrum in the AM-FMCW radar receiver before and after the $5^{\text {th }}$ order Chebychev low-pass filter, where $\Delta \omega$ is the beat frequency produced by a unique target at a distance of 200 meters. The $\omega_{d} \pm \Delta \omega$ components are mixing components due to the amplitude modulation introduced in the transmitter.
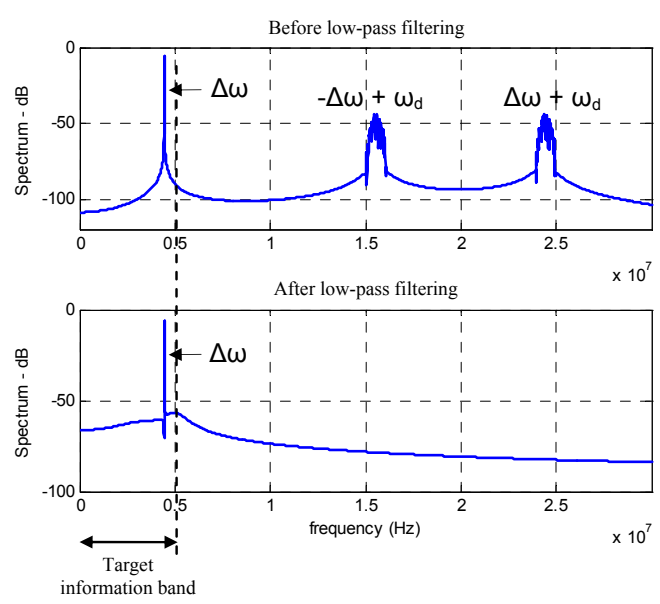

Fig. 9 Spectrum in the AM-FMCW radar receiver

As in any FMCW radar, to obtain the Doppler frequency produced by a moving target, and thus know its velocity, it is needed to repeat the linear frequency sweep several times. The amplitude of the target beat frequency will follow this Doppler frequency.

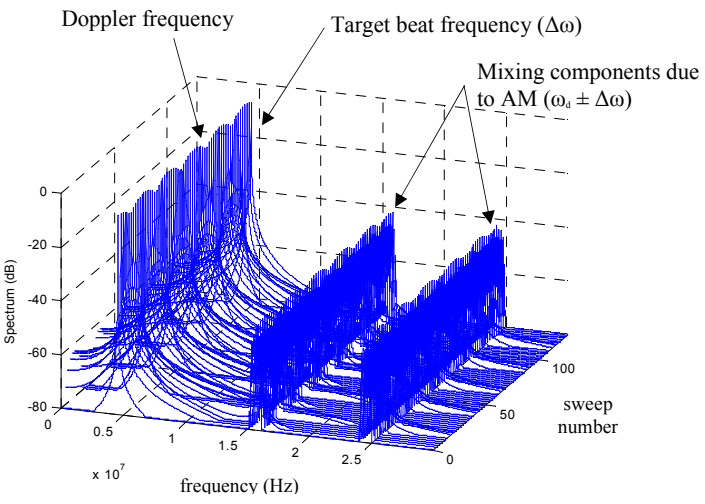

Fig. 10 Several sweeps performance to measure the Doppler frequency

Fig. 10 represents, in the same plot, the 128 sweeps performed for the case of one target at an initial distance of 200 meters approaching the radar at a velocity of $5 \mathrm{~m} / \mathrm{s}$. Note that the present mixing components would be filtered out by the low-pass filter.

\section{AM Data Receiver}

As depicted in Fig. 5, a loop structure is utilized to synchronize the received and the generated sweeps in the AM data receiver. The delay between them is adjusted until the frequency difference reaches a desired intermediate frequency ( $I F$ ). When this condition is achieved, in the mixer output spectrum one intermediate component will appear plus two data side bands at $I F \pm \omega_{d}$, as shown in Fig. 11. Then, by filtering one of the side bands and simple demodulation the data can be recovered.

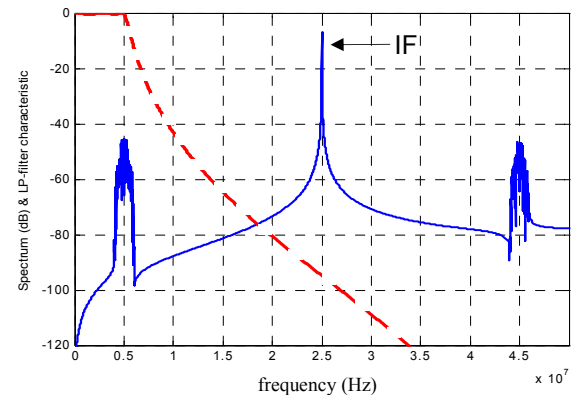

Fig. 11 Spectrum in the AM Data Receiver after the synchronization loop, and AM Data Receiver low-pass filter characteristic (doted line)

\section{CONCLUSIONS}

With the AM-FMCW system the conjunction of two functions, radar and communication, is achieved. With the proposed solution it is possible to (1) measure the range to targets, (2) measure the velocity of targets, and (3) communicate information using a single signal and a single system.

An advantage of this technique is that actual FMCW radar networks could be easily modified to send information as well. Furthermore, there is a broad bandwidth available to send information because of the large bandwidths available to radar systems and the large frequency sweep of the FMCW radar. This broad bandwidth also offers the possibility to implement more complex communication systems, such as multi-channel or frequency hopping systems.

A expression has been derived for the data carrier frequency that avoids degradation of the radar performance. As mentioned the demodulation in the data receiver is not straightforward, and requires synchronization between the sweeps.

Finally, if duplex communication would be required two AM-FMCW radars working at different frequencies must be applied.

\section{REFERENCES}

[1] M. Skolnik, Introduction to Radar Systems, 2nd ed., Ed. McGraw-Hill, 1985. Chapter 3: CW and FM Radar.

[2] Ad van Heijningen, Johan Janssen, Anne de Zeeuw, ViApache: Traffic Observation using Defence Technology, 6th European Congress of ITS, Aalborg, Denmark, 18-20 June 2007.

[3] M. Skolnik, Introduction to Radar Systems, chapter 4, 2nd ed., Ed. McGraw-Hill, 1985. Chapter 4: MTI and Pulse Doppler Radar.

[4] A.G. Stove, "Linear FMCW radar techniques," in IEE Proceedings-F, Vol. 139, No. 5, October 1992.

[5] N. S. Tzannes, Communication and Radar Systems, Ed. Authors Choice Press, USA, 2000. 